NOTE

\title{
Source and detector polarization effects on radiographic film dosimetry
}

\author{
Tsang Cheung ${ }^{1}$, Peter K N Yu ${ }^{1}$ and Martin J Butson ${ }^{1,2}$ \\ ${ }^{1}$ City University of Hong Kong, Department of Physics and Materials Science, Kowloon Tong, \\ Hong Kong, People's Republic of China \\ 2 Illawarra Cancer Care Centre, Department of Medical Physics, Crown St, Wollongong NSW \\ 2500, Australia \\ E-mail: butsonm@iahs.nsw.gov.au
}

Received 1 July 2003

Published 7 November 2003

Online at stacks.iop.org/PMB/48/N329

\begin{abstract}
Kodak X-Omat V radiographic film has been tested for its polarization properties when using polarized and non-polarized light sources and detectors. The radiographic film has been shown to produce a relatively small but not negligible (less than 5\%) variation in $90^{\circ}$ cycles in measured optical density within the visible spectrum when the light source is fully linear polarized and the film is rotated through $360^{\circ}$ angle. Negligible variations are seen when the detector is linearly polarized. If both light source and detector are linearly polarized, variations in measured optical density can reach $35 \%$ when the film is rotated through $360^{\circ}$ angle. This seems to be due to variations in the degree and axis of rotation in polarized light caused by the radiographic film and is independent of exposure level as the intensity of variation in absolute optical density is relatively the same for all film exposures. We recommend that a polarization test be performed on a densitometry system to establish the extent of its polarization properties before accurate dosimetry is performed with radiographic film.
\end{abstract}

\section{Introduction}

Radiographic film has been a popular dosimetry tool in radiotherapy for at least 50 years. This has been due to advantageous characteristics of film such as the fact that it provides a permanent record and 2D film plane dosimetric information from a single exposure. Radiographic film is also an integrating dosimeter with good spatial resolution, a relatively short exposure time and it is economical (Hale et al 1994, Robar and Clark 1999, Yeo et al 1997, Williamson et al 1981, Weltens et al 1998). Radiographic film also has disadvantages in its use, which need to be corrected for or at least taken into account during dosimetry. These include film artefacts, inter- and intra-film batch variations, sensitometric non-linearity, 
chemical processing condition dependence and film orientation dependence (Wang et al 2002, Blendl and Buhr 2001, Suchowerska et al 1997, 1999, 2001, Sykes et al 1999). Another factor, which can influence dosimetric results, is the polarization properties of the radiographic film and its effects when using polarized light sources and polarized detectors. It has been noted that radiochromic film produces polarization effects during dosimetry (Butson et al 2003). With respect to light, polarization is said to occur if there is a restriction of the vibrations of the magnetic or electric field vector to a single plane. In a beam of electromagnetic radiation, the polarization direction is the direction of the electric field vector (with no distinction between positive and negative as the field oscillates back and forth). The polarization vector is always in the plane at right angles to the beam direction. When the end point of the electric vector of a polarized light source is viewed along the direction of light propagation, it moves along a straight line if the light is linearly polarized, along a circle if the light is circularly polarized and along an ellipse if it is elliptically polarized. Some analysing light sources are polarized to some degree whereas some are not. Light sources diffraction grating photo spectrometers produce polarized light sources due to the nature of grating. He-Ne lasers can produce two orthogonally polarized modes in their densitometry light sources (Demspey et al 2000). Most top quality densitometers/scanners use polarization filters in their systems as this eliminates optical density (OD) measurement variations for reflectance measurements between glossy and matte images (Gretag MacBeth 2002). This note investigates the variations in measured optical density seen using radiographic film and polarized light sources and polarized detector systems.

\section{Materials and methods}

Measurements to assess the effects of polarized light sources and detectors have been made using a Gammex X-rite 331 densitometer. The densitometer has an optical density measuring range of $0-4.0$ OD units with an accuracy of \pm 0.02 and reproducibility of \pm 0.01 OD units. It uses a white light source and a visible photo diode detector. Tests were performed to analyse the initial polarization properties of this system using linearly polarized sheets. Results showed that a small degree of polarization was present with a variation of approximately $3 \%$ in measured optical density present when a fully linear polarized sheet was rotated through its axis of polarization.

The film used was Kodak X-Omat $\mathrm{V}$ radiographic film which was exposed to various absorbed doses ranging from $0 \mathrm{~Gy}$ up to $0.8 \mathrm{~Gy}$ in $0.1 \mathrm{~Gy}$ intervals. The films were processed in an automatic X-Omat processor before analysis. Each film was attached to a calibrated angular protractor, which could easily rotate $360^{\circ}$ within the constructed densitometer. The polarizers used were Coherent Scientific linear polarized sheets with size $5 \mathrm{~cm} \times 5 \mathrm{~cm}$. When two sheets were placed together and rotated to minimum transmission, approximately $0.2 \%$ transmission was observed using this densitometer.

To test the polarization properties of the X-Omat $\mathrm{V}$ radiographic film, four tests were performed. These were, (1) effects of film rotation with relatively unpolarized (3\%) lights and detectors, (2) effects on film dosimetry with a polarized light source, (3) effects of a polarized detector and (4) effects of both light source and detector polarized. Test 1 was performed by rotating the $\mathrm{X}-\mathrm{Omat} \mathrm{V}$ radiographic film through $360^{\circ}$ rotation and measuring optical density results in $10^{\circ} / 5^{\circ}$ intervals depending on position near maxima/minima. Test 2 was performed by fixing a linear polarizer sheet to the light source, thus producing a linear polarized light source with unpolarized detector. The film was then rotated through $360^{\circ}$ and optical density measurement made in $10^{\circ}$ steps. Test 3 was performed by fixing the linear polarized sheet to the detector, thus producing a linear polarized detector with unpolarized light source. Again 


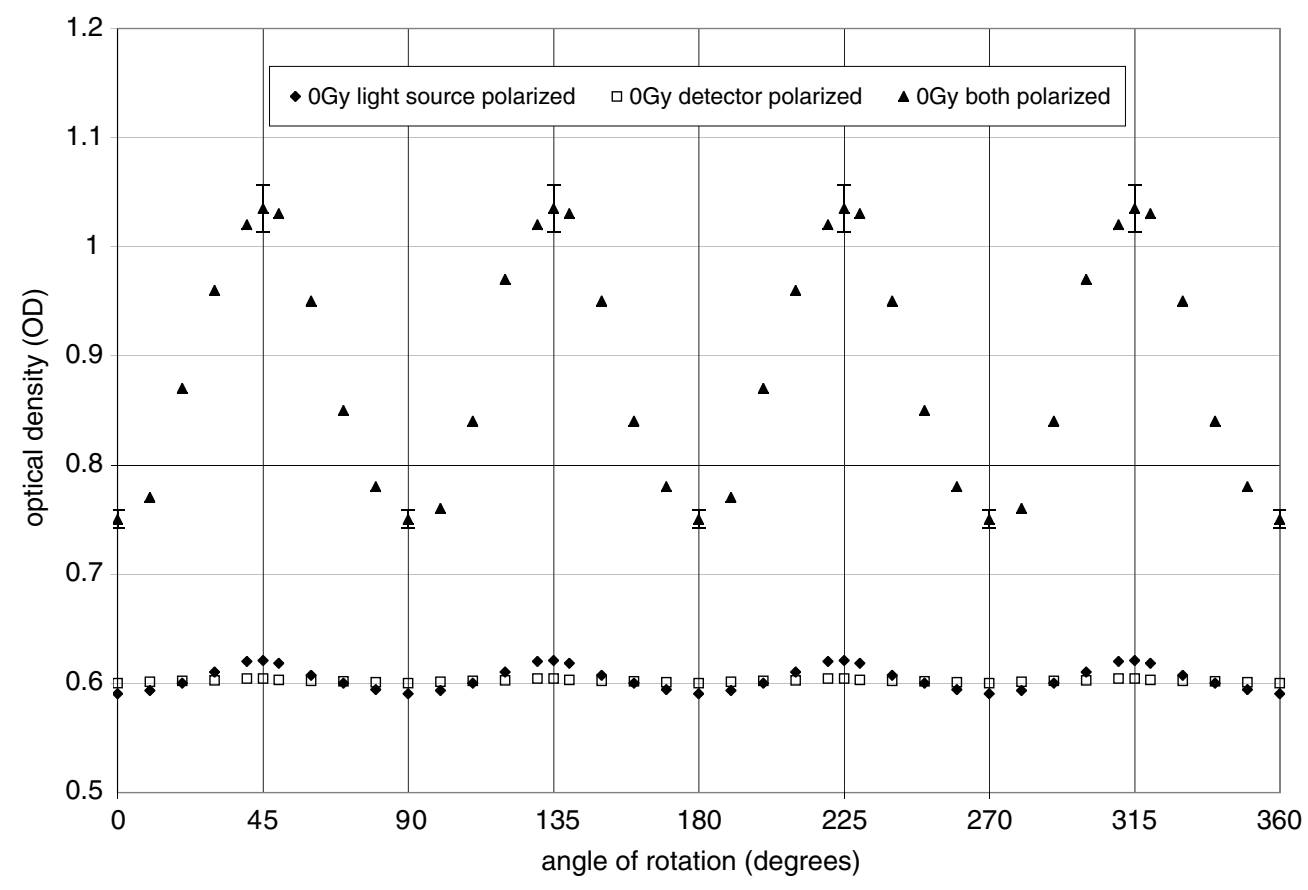

Figure 1. Variations in optical density as a function of angle of rotation of an unirradiated X-Omat $\mathrm{V}$ radiographic film. Results are for polarized light source, polarized detector and both polarized.

the X-Omat V film was inserted and optical density measurements were made through $360^{\circ}$ of rotation in $10^{\circ}$ intervals. Test 4 was performed by attaching linear polarized sheets to both the light source and the detector. The orientation of the polarizers was made to produce a maximum intensity output, thus the linear polarization was in the same plane for the two sheets. The X-Omat V radiographic film was then inserted between the polarizers and measurements were made for optical density for $360^{\circ}$ rotation. No corrections were performed on results to account for the $3 \%$ initial polarization of the detector system.

\section{Results and discussion}

Figure 1 shows the effects of polarized light sources, polarized detectors and both polarized for unirradiated X-Omat V radiographic film. The initial absolute optical density for the film was approximately 0.16 at the $0^{\circ}$ position. We have defined the $0^{\circ}$ position as the position for minimum intensity output from the film when polarized conditions are applied. Error bars are only shown at $90^{\circ}$ intervals to improve the clarity of the figures, however, they are representative of all measurements and are one standard deviation for five measurements over two pieces of film in each case. Results show that a small polarization effect occurs when only the light source is polarized with a $5 \%$ variation seen with a cyclic response over a $90^{\circ}$ rotation. Negligible effects were seen when just the detector was polarized. When both light source and detector were polarized, a larger variation in OD was measured with an approximate variation of $37 \%$ seen. Similar normalized results were seen for irradiated X-Omat V film at all doses measured and are represented by results in figure 2 which is for 60 cGy applied dose. Results show that an approximate $1 \%$ variation in optical density is seen with polarized light source and negligible effects when the detector is polarized. The normalized variation for the 




Figure 2. Variations in optical density as a function of angle of rotation of a X-Omat V radiographic film irradiated to $60 \mathrm{cGy}$. Results are for polarized light source, polarized detector and both polarized.

Table 1. Maximum variations in optical density (OD) caused by polarized light sources, polarized detectors and both at various dose levels.

\begin{tabular}{llllll}
\hline & \multicolumn{5}{c}{ Dose (cGy) } \\
\cline { 2 - 6 } Device polarized & 0 & 20 & 40 & 60 & 80 \\
\hline Light source & 0.03 OD & 0.03 & 0.02 & 0.02 & 0.02 \\
Detector & 0.00 & 0.00 & 0.01 & 0.01 & 0.01 \\
Both & 0.28 & 0.28 & 0.27 & 0.26 & 0.26 \\
\hline
\end{tabular}

irradiated radiographic film was less, being an approximate $12 \%$ variation. Table 1, however, shows that the variations seen for all films are similar on an absolute optical density scale. Thus, it appears that the variations seen are independent of exposure level within 0 cGy-80 cGy and thus a larger percentage effect is seen for lower dosed films. The base material is the common layer for all films whereas the silver halide image layer varies with level of irradiation delivered. Radiographic film can vary in thickness from 0.2 to $0.38 \mathrm{~mm}$. The base is the thickest component, typically being $0.18 \mathrm{~mm}$ thick. Its purpose is to support the film emulsion, while permitting processing chemicals access to the active components in the emulsion. The base often has a blue tint to reduce the glare when viewed on a light box. The larger variation seen with angle of rotation in both data sets is assumed to be due to light scattering produced by the radiographic film between the linearly polarized light source and detector as would be the case for many plastic-based thin films. This causes a certain degree of non-polarization before striking the polarized detector. Table 2 shows results for OD output when the initial OD output for the fully polarized system produces a minimum light output, i.e. $45^{\circ}$ angle of rotation of the radiographic film and the analyser (detector polarization) is 
Table 2. Variations in measured optical density with angle of rotation of detector polarization with film angle of rotation set to minimum initial transmission $\left(45^{\circ}\right)$.

\begin{tabular}{rl}
\hline Film angle of rotation $\left(^{\circ}\right)$ & Optical density (OD) \\
\hline 0 & 1.15 \\
15 & 1.13 \\
30 & 1.10 \\
45 & 1.10 \\
60 & 1.13 \\
75 & 1.18 \\
90 & 1.23 \\
105 & 1.4 \\
120 & 1.51 \\
135 & 1.52 \\
150 & 1.45 \\
165 & 1.3 \\
180 & 1.21 \\
\hline
\end{tabular}

rotated through various angles. Results show that cyclic variation in light output occurs but the absolute intensity is decreased at all angles. Thus, the film material has partially scattered the incident light producing a semi-polarized source before entering the polarized detector. Some densitometers, scanners or photo spectrometers may have polarized light sources or detector systems. Polarization is used as a tool for colour matching and image enhancement in scanners for eliminating interference caused by reflections and associated optic degradation. The extent of the polarization properties of the light source and detector will in turn affect the quantity of the variations in OD seen with film angular rotation. Thus, we would recommend that a test for polarization using a polarizer film should be conducted to assess this property for a densitometer before film analysis is performed to improve accuracy of dosimetry with radiographic film.

\section{Conclusion}

$\mathrm{X}$-Omat $\mathrm{V}$ radiographic film has been tested for polarization properties and found to be minimally polarized. This in effect produces a small variation in measured optical density as a function of angle of rotation if a polarized light source or polarized detector system was used. If, however, a densitometer system had both light source and detector polarized, X-Omat $\mathrm{V}$ radiographic film can significantly (up to $37 \%$ ) change the measured optical density by producing a variation in the degree and axis of rotation of polarization in the transmitted light as a function of the angle of rotation of the film. The polarization properties of a densitometer light source and detector should be tested before quantitative dosimetry is performed with $\mathrm{X}$-Omat $\mathrm{V}$ radiographic film to improve the accuracy available with this dosimetry film.

\section{Acknowledgment}

This work has been fully supported by a grant from the Research Grants Council of HKSAR, China (project no CityU 1012/01P).

\section{References}

Blendl C and Buhr E 2001 Comparison of light and x-ray sensitometric responses of double-emulsion films for different processing conditions Med. Phys. 28 2420-6 
Butson M J, Yu K N, Cheung T and Metcalfe P E 2003 Radiochromic film for medical radiation dosimetry Mater. Sci. Eng. 41 61-120

Dempsey J F, Low D A, Mutic S, Markman J, Kirov A S, Nussbaum G H and Williamson J F 2000 Validation of a precision radiochromic film dosimetry system for quantitative two-dimensional imaging of acute exposure dose distributions Med. Phys. 27 2462-75

Gretag Macbeth Colour Control Centre 2002 Applied Densitometry Technical Manual

Hale J I, Kerr A T and Shragge P C 1994 Calibration of film for accurate megavoltage photon dosimetry Med. Dosim. $1943-6$

Robar J L and Clark B G 1999 The use of radiographic film for linear accelerator stereotactic radiosurgical dosimetry Med. Phys. 26 2144-50

Suchowerska N, Davison A, Drew J and Metcalfe P 1997 The validity of using radiographic film for radiotherapy dosimetry Australas. Phys. Eng. Sci. Med. 20 20-6

Suchowerska N, Hoban P, Butson M, Davison A and Metcalfe P 2001 Directional dependence in film dosimetry: radiographic and radiochromic film Phys. Med. Biol. 46 1391-7

Suchowerska N, Hoban P, Davison A and Metcalfe P 1999 Perturbation of radiotherapy beams by radiographic film: measurements and Monte Carlo simulations Phys. Med. Biol. 44 1755-65

Sykes J, James H and Williams P 1999 How much does film sensitivity increase at depth for larger field sizes Med. Phys. 26 329-30

Wang Y, Cross P and Zealey W 2002 An investigation into the source of low energy scattered radiation of significance in film dosimetry Australas. Phys. Eng. Sci. Med. 25 139-43

Weltens C, Huyskens D, Dutreix A and Schueren van der E 1998 Assessment of dose inhomogeneities in clinical practice by film dosimetry Radiother. Oncol. 49 287-94

Williamson J, Khan F and Sharma S 1981 Film dosimetry of megavoltage photon beams: a practical method of isodensity to isodose curve conversion Med. Phys. 8 94-8

Yeo I J, Wang C K and Burch S E 1997 A filtration method for improving film dosimetry in photon radiation therapy Med. Phys. 24 1943-53 